\title{
盐度骤变对花鲇血清及肝脏代谢酶、 抗氧化酶活力及皮质醇含量的影响
}

\author{
王孝杉 ${ }^{1}$ ，方 秀 ${ }^{2}$, 彭士明 $^{1}$ ，汪 睛 $^{2}$ ，施兆鸿 ${ }^{1}$ \\ (1. 中国水产科学研究院东海水产研究所, 上海 $200090 ; 2$. 福建闽威实业股份有限公司, 福建福鼎 355200 )
}

\begin{abstract}
摘 要: 为探讨花鲇 (Lateolabrax maculatus) 血清和肝脏中代谢酶和抗氧化酶活力以及血清皮质醇含量对盐 度骤变的响应,设置两种盐度变化处理方式: 1) 盐度 18 骤降至 0 并持续 $7 \mathrm{~d}$, 然后再骤升至盐度 33 并持续 7 $\mathrm{d} ; 2)$ 盐度 18 骤升至 33 并持续 $7 \mathrm{~d}$, 然后再骤降至盐度 0 并持续 $7 \mathrm{~d}$ 。分别在盐度骤升和骤降处理后 $0 、 0.5 、 1$ 、 $3,7 \mathrm{~d}$ 时取样,测定花鲇血清和肝脏中碱性磷酸酶、酸性磷酸酶、谷丙转氨酶、谷草转氨酶、超氧化物歧化酶、 过氧化氢酶、谷胱甘肽过氧化物酶活力以及血清皮质醇含量。结果显示, 在盐度大幅度骤变条件下, 花鲇代谢 酶、抗氧化酶活力以及皮质醇含量都发生变化。在大部分骤变条件下,酶活力和皮质醇含量均随时间推移呈 现先升高后降低的变化趋势, 表明花鲇在盐度骤变时产生了应激反应; 但在处理后 $3 \sim 7 \mathrm{~d}$, 酶活力和皮质醇含 量可恢复至处理前水平, 表明花鲇对盐度骤变具有良好的耐受性和适应性。研究结果表明,本实验条件下,花 鲇在遭遇养殖环境中盐度骤降变化时, 可以较快地恢复, 说明该品种对低盐环境有较强的适应能力。
\end{abstract}

关键词: 花鲇; 盐度骤变; 代谢酶活力; 抗氧化酶活力; 皮质醇含量

中图分类号：S917 文献标志码：A

海水鱼类对盐度的耐受性和适应性与渗透 压调节能力和离子调节酶活力有关, 在对盐度的 适应过程中, 鱼类的生长、代谢、免疫、性腺发育 等生理功能都会受到影响 ${ }^{[1]}$ 。环境中盐度变化 可引起鱼类多种生理应激反应, 导致产生过量的 活性氧分子 ${ }^{[2]}$, 影响到鱼类的存活、代谢及免疫 防御功能 ${ }^{[3]}$ 。鱼类机体中抗氧化系统的抗氧化 酶主要包括超氧化物歧化酶 (SOD)、过氧化氢酶 (CAT)、谷胱甘肽过氧化物酶 (GSH-Px) 等, 这 3 种酶协同作用, 有效防护了活性氧对机体的损 伤 ${ }^{[4]}$, 在清除活性氧自由基方面发挥着重要作 用 ${ }^{[5]}$ 。生物机体的部分新陈代谢活动是依赖不 同磷酸酶来催化完成分子的磷酸化和去磷酸 化 ${ }^{[6]}$ 。其中, 酸性磷酸酶 (ACP) 和碱性磷酸酶 (AKP) 是衡量受盐度胁迫后机体免疫机能和健
康状况的重要指标 ${ }^{[7]}$ 。而能反映受盐度胁迫后 机体应激程度的转氨酶主要存在于血液和肝脏 等组织中,一般认为谷丙转氨酶 (GPT) 和谷草转 氨酶(GOT) 是氨基酸代谢过程中两个重要的氨 基转移酶 ${ }^{[8]}$ 。同样, 皮质醇( COR) 不仅对应激反 应和糖代谢有调控功能, 对渗透压也有调节作 用, 能通过增加氯细胞数量和提高 $\mathrm{Na}^{+}-\mathrm{K}^{+}-\mathrm{ATP}$ 酶活力来增强机体耐盐性 ${ }^{[9]}$ 。可见, 抗氧化酶和 代谢酶活力以及皮质醇含量对研究盐度胁迫条 件下的鱼类适应性和耐受性具有重要的意义。

花鲈 (Lateolabrax maculatus) 是我国一种重 要的经济鱼类, 其盐度适应范围广, 在从淡水到 高盐海水等不同盐度的水环境中均能存活生 长 $^{[10]}$ 。有关盐度变化对花鲈生长及生理生态影 响的研究, 20 世纪 90 年代主要集中在盐度对其

收稿日期: $2020-02-28$

基金项目：现代农业产业技术体系专项资金(CARS-47);农业农村部“一带一路”沿线热带国家水产养殖科技合作 项目 (水产经济动物养殖与模式构建技术输出交流 2019); 广西科技基地和人才专项 (桂科 AD17195024)

作者简介：王孝杉 (1986一), 男, 助理研究员, 主要从事海水鱼类繁育研究。E-mail: wangxs@ ecsf. ac. cn 通信作者：彭士明,研究员。E-mail:pengsm@ecsf. ac.cn 
胚胎和仔鱼生长发育的影响方面 ${ }^{[11-12]} ; 21$ 世纪 以来开展了盐度及饥饿胁迫相关实验和研 究 $^{[13-16]}$; 近 10 年来研究则主要集中在盐度胁迫 对鱼类生长性能、生理生态、组织结构、基因表达 的影响等方面 ${ }^{[17-25]}$ 。为评估花鲈在养殖环境中 对盐度骤变的耐受性和适应性, 本实验设置盐度 骤降再骤升和盐度骤升再骤降两种处理方式, 观 察其肝脏代谢酶和抗氧化酶活力及皮质醇含量 变化情况, 旨在得出花鲇肝脏和血清中抗氧化酶 和代谢酶活力以及皮质醇含量在盐度单一骤变 及连续骤变过程中的响应规律, 并为花鲈应对盐 度骤变的生理机制研究提供基础资料。

\section{1 材料与方法}

\section{1 实验用鱼与实验条件}

实验于 2018 年 9 月在东海水产研究所福鼎 试验基地进行。实验用鱼取自沙埕港内的福建 闽威实业股份有限公司养殖网箱, 选取大小一 致、活力较好的花鲇个体, 平均体长 ( $23.23 \pm$ $1.13) \mathrm{cm}$, 平均体质量 $(203.27 \pm 26.55) \mathrm{g}$ 。鱼 运抵后先在水温 $(26.0 \pm 1.0)^{\circ} \mathrm{C}$ 、盐度 20 的室 内水泥池中暂养 $14 \mathrm{~d}$ 。暂养密度为 30 尾 $\cdot \mathrm{m}^{-3}$, 每天 8:00 和 17:00 饱食投喂日本林兼产业株式 会社产 “虎豚” 牌 2 号配合饲料。不间断向水中 充气, 每天上午在投饲 $2 \mathrm{~h}$ 后换同盐同温水 $50 \%$, 同时吸去底部污物及残饵。实验用水为过滤沉 淀处理后的天然海水。

\section{2 实验设计}

实验在直径 $1.2 \mathrm{~m}$ 、深 $0.8 \mathrm{~m}$ 的圆形玻璃纤 维缸中进行。实验设计见表 1 , 盐度骤变处理分 $A 、 B$ 两组: $A$ 组从盐度 18 先骤降至盐度为 0 的淡 水并持续 $7 \mathrm{~d}$, 以 $\mathrm{A}-\mathrm{I}$ 表示; 然后再骤升至盐度 33 的水体中持续 $7 \mathrm{~d}$, 以 $\mathrm{A}-\mathrm{II}$ 表示; $\mathrm{B}$ 组从盐度 18 骤 升至盐度 33 的水体并持续 $7 \mathrm{~d}$,以 B-I 表示; 然后 再骤降到盐度为 0 的淡水中并持续 $7 \mathrm{~d}$, 以 B-II 表 示。另设盐度 18 养殖水体处理组为对照组 $(\mathrm{C}$ 组)。盐度 0 的养殖水采用曝气后的淡水; 盐度 33 的养殖水采用盐度 18 的过滤沉淀后的天然海 水添加海水晶配置, 配制好的养殖水稳定 $48 \mathrm{~h}$ 后 使用。用折光式 PAL-03S 盐度计 (爱拓牌, 南京 晓晓仪器设备有限公司) 监测养殖水的盐度。每 组均设 3 个重复,每个重复 30 尾鱼。实验期间的 管理与暂养期间一致, 每天观察鱼的活动和摄食
状况。

表 1 盐度骤变的实验设置

Tab. 1 Experiment design of abrupt salinity changes

\begin{tabular}{cccc}
\hline \multirow{2}{*}{$\begin{array}{c}\text { 实验时间/阶段 } \\
\text { Experiment time/phase }\end{array}$} & \multicolumn{2}{c}{$\begin{array}{c}\text { 各组别处理盐度 } \\
\text { Salinity treatment } \\
\text { of different groups }\end{array}$} \\
\cline { 2 - 4 } & A & B & $\begin{array}{c}\text { 对照组 }(\mathrm{C}) \\
\text { Control }(\mathrm{C})\end{array}$ \\
\hline $0 \mathrm{~d}$ & 18 & 18 & 18 \\
$0 \sim 7 \mathrm{~d} /$ 第 I 阶段 Phase I & 0 & 33 & 18 \\
$7 \sim 14 \mathrm{~d} /$ 第 II 阶段 Phase II & 33 & 0 & 18 \\
\hline
\end{tabular}

\section{3 取样和样品检测}

第 I 和第 II 阶段开始后分别于 $0 、 0.5 、 1 、 3 、 7$ $d$ 时取样, 每个平行组每次取 3 尾鱼。取样前禁 食半天, 用 3-氨基苯甲酸乙酯甲基磺酸盐( MS$222,200 \mathrm{mg} \cdot \mathrm{L}^{-1}$ ) 将鱼麻醉后, 用 $1 \mathrm{~mL}$ 注射器 (预冷) 从尾部静脉抽取血液置人 $5 \mathrm{~mL}$ 离心管 中, 离心管在 $4{ }^{\circ} \mathrm{C}$ 下静置 $12 \mathrm{~h}$, 采用安信 AXTGL16M 型低温离心机 $2500 \mathrm{r} \cdot \mathrm{min}^{-1}$ 条件下 离心 $5 \mathrm{~min}$, 取上清液, 置于 $2 \mathrm{~mL}$ 离心管中放在 $-20{ }^{\circ} \mathrm{C}$ 冰箱中保存, 用于检测血清 AKP、ACP、 GPT、GOT 活力和 COR 含量。取完血样后将鱼置 于冰盘上解剖, 取出肝脏, 用预冷生理盐水冲洗 后再用吸水纸吸干, 并置于 $2 \mathrm{~mL}$ 的离心管中在 $-20{ }^{\circ} \mathrm{C}$ 冰箱中保存, 用于检测 SOD、CAT、GSH-Px 活力以及 AKP、ACP、GPT、GOT 活力。测定前, 先 将肝脏在匀浆介质 $\left(\mathrm{pH} 7.4,0.01 \mathrm{~mol} \cdot \mathrm{L}^{-1}\right.$ Tris$\mathrm{HCl}, 0.0001 \mathrm{~mol} \cdot \mathrm{L}^{-1}$ EDTA-2Na, 0. $01 \mathrm{~mol}$ • $\mathrm{L}^{-1}$ 蔗糖, $0.8 \% \mathrm{NaCl}$ ) 中剪碎, 用沪析 HR-6 型匀 浆机( $15000 \mathrm{r} \cdot \mathrm{min}^{-1}$ ) 研磨制成匀浆。在 4 ${ }^{\circ} \mathrm{C} 、 1500 \mathrm{r} \cdot \mathrm{min}^{-1}$ 条件下离心 $5 \mathrm{~min}$ 。根据需 要, 取上清液稀释后进行酶活力及总蛋白测定。 总蛋白采用考马斯亮兰法 ( 南京建成试剂盒) 进 行测定。酶活力用南京建成试剂盒检测, 按照试 剂盒说明书进行操作。

\section{4 数据统计与分析}

实验结果用 SPSS 16.0 软件进行统计与分 析。采用单因素方差分析 ( one-way ANOVA) 和 独立样本 $t$ 检验对 3 个组别的差异性进行分析, 当 $P<0.05$ 时表示差异具有显著性, 文中数据均 以平均值 \pm 标准差 $($ mean $\pm \mathrm{SD})$ 表示。用 Excel 2010 软件作图。 


\section{2 结果与分析}

\section{1 盐度骤变对花鲇活动和摄食的影响}

在阶段 I 中, A 组从盐度 18 骤降至 0 ,鱼游 动正常, 与 $\mathrm{C}$ 组无差异; 但盐度骤变后出现停食 现象,到 $3 \mathrm{~d}$ 时才开始恢复摄食。 $\mathrm{B}$ 组从盐度 18 骤升至 33 , 鱼活动也正常, 到 $6 \mathrm{~d}$ 时开始恢复摄 食。

在阶段 II 中,盐度骤变首日, $A 、 B$ 组鱼活动 均表现为经常卧底、游动迟缓, $3 \mathrm{~d}$ 起各组鱼游动 能力逐渐恢复, $6 \mathrm{~d}$ 起游动状态与 $\mathrm{C}$ 组无差异; $7 \mathrm{~d}$ 内均未摄食。

\section{2 盐度骤变对花鲇血清代谢酶活力的影响}

如图 1 所示, 在 A-I 阶段中, A 组血清中 $\mathrm{AKP} 、 \mathrm{ACP} 、 \mathrm{GPT}$ 和 GOT 活力均出现上升。其中, $\mathrm{AKP}$ 仅在 $0.5 \mathrm{~d}$ 与 $\mathrm{C}$ 组间差异显著 $(P<0.05)$; ACP、GPT 活力在 $0.5 \sim 3 \mathrm{~d}$ 时与 $\mathrm{C}$ 组间差异显著 $(P<0.05)$; GOT 活力在 $3 \mathrm{~d}$ 时与 $\mathrm{C}$ 组间差异显 著 $(P<0.05)$; 随后, 各代谢酶活力下降至与 $\mathrm{C}$ 组
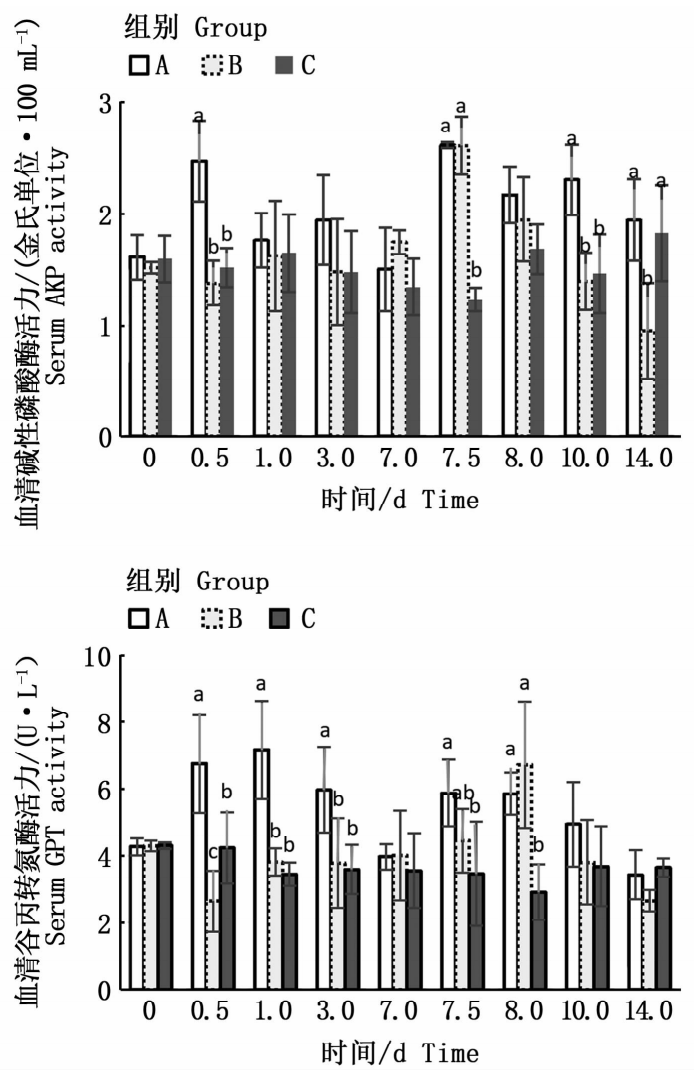

间无显著性差异 $(P>0.05)$ 。在 A-II 阶段, 盐度 从 0 骤变至 $33, \mathrm{~A}$ 组各代谢酶活力均又出现上 升。其中, $\mathrm{AKP}$ 在处理后 $0.5 \mathrm{~d}$ 和 $3 \mathrm{~d}$ 时与 $\mathrm{C}$ 组 间差异显著 $(P<0.05)$; ACP 在 $0.5 \mathrm{~d}$ 时与 $\mathrm{C}$ 组 间差异显著 $(P<0.05)$; GPT 和 GOT 在 $0.5 \sim 1 \mathrm{~d}$ 时与 $\mathrm{C}$ 组间差异显著 $(P<0.05)$; 随后各指标下 降至与 $\mathrm{C}$ 组间无显著性差异 $(P>0.05)$ 。

B-I 阶段, B 组 AKP 和 ACP 活力与 $\mathrm{C}$ 组间无 显著性差异 $(P>0.05)$ 。而 GPT 和 GOT 活力分 别在处理后 $0.5 \mathrm{~d}$ 和 $0.5 \sim 3 \mathrm{~d}$ 时出现下降, 并与 C 组间有显著性差异 $(P<0.05)$; 随胁迫时间延 长, GPT 在 $1 \mathrm{~d}$ 时恢复至与 $\mathrm{C}$ 组间无显著性差异 $(P>0.05)$, GOT 则在 $7 \mathrm{~d}$ 时恢复到与 $\mathrm{C}$ 组间无 显著性差异 $(P>0.05)$ 。在随后 B-II（盐度 33 骤 降至 0$)$ 阶段, B 组 AKP $0.5 \mathrm{~d}$ 时先显著上升 $(P<$ $0.05)$, 随后开始下降, 至 $7 \mathrm{~d}$ 时降至显著低于 $\mathrm{C}$ 组 $(P<0.05)$; $\mathrm{ACP}$ 活力在 $0.5 \sim 3 \mathrm{~d}$ 时均与 $\mathrm{C}$ 组 间有显著性差异 $(P<0.05)$; 至 $7 \mathrm{~d}$ 时恢复至无 显著性差异 $(P>0.05)$ 。GPT 活力在 $1 \mathrm{~d}$ 时与 $\mathrm{C}$
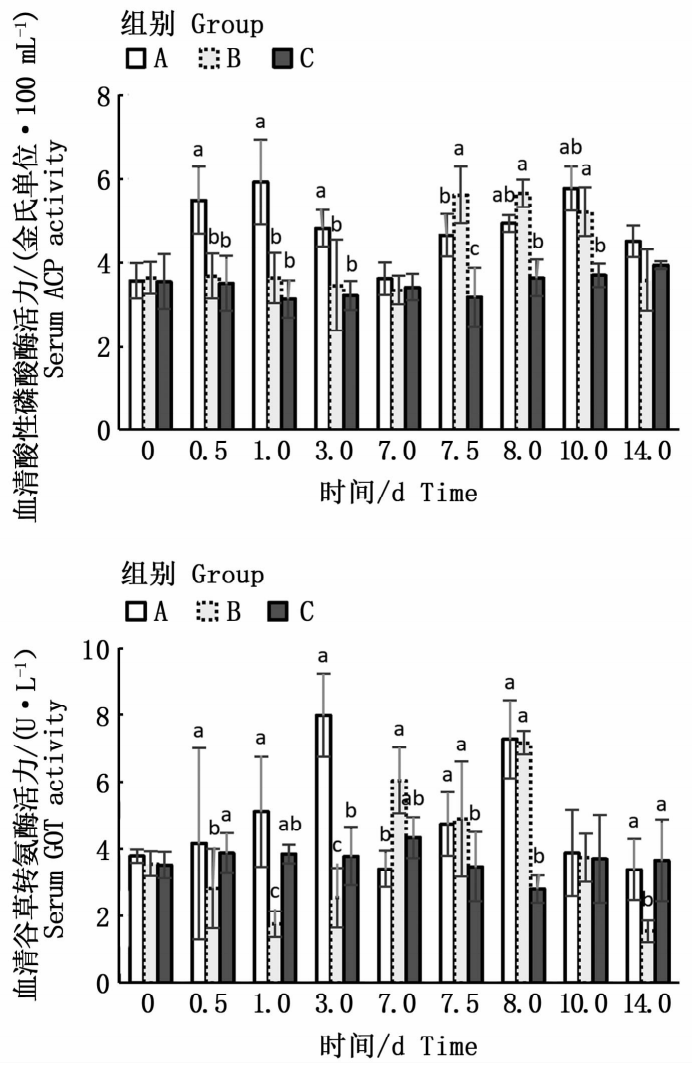

图 1 盐度骤变对花鲇血清代谢酶活力的影响

Fig. 1 Impact of abrupt salinity changes on activity of metabolic enzymes in serum of Lateolabrax maculatus 注: 不同字母表示同一时间不同组间差异显著 $(P<0.05)$

Note: Different letters indicate significant differences between different groups at the same time $(P<0.05)$ 
组呈显著性差异 $(P<0.05)$, 随后下降至无显著 性差异 $(P>0.05)$; GOT 活力出现先升后降的变 化, $0.5 \mathrm{~d}$ 和 $1 \mathrm{~d}$ 时显著高于 $\mathrm{C}$ 组 $(P<0.05), 7 \mathrm{~d}$ 时又下降至显著低于 $\mathrm{C}$ 组 $(P<0.05)$ 。

\section{3 盐度骤变对花鲇血清皮质醇含量的影响}

如图 2 所示,在 A-I 阶段中, A 组血清中 COR 含量出现上升, 在 $0.5 \sim 3 \mathrm{~d}$ 时均显著高于 $\mathrm{C}$ 组 $(P<0.05) ; 7 \mathrm{~d}$ 时下降至与 $\mathrm{C}$ 组间无显著性差异 $(P>0.05)$ 。A-II 阶段, A 组 COR 含量在 $0.5 \sim 1$ $\mathrm{d}$ 时显著高于 $\mathrm{C}$ 组 $(P<0.05)$; 随后 $3 \sim 7 \mathrm{~d}$ 时降 至与 $\mathrm{C}$ 组间无显著性差异 $(P>0.05)$ 。

在 B-I 阶段中, 实验 $3 \mathrm{~d}$ 内 B 组 COR 含量与 C 组间无显著性差异 $(P>0.05), 7 \mathrm{~d}$ 时 $\mathrm{B}$ 组与 $\mathrm{C}$ 组间有显著性差异 $(P<0.05)$ 。B-II 阶段中, B 组 $\mathrm{COR}$ 含量先升高后降低, 在 $1 \mathrm{~d}$ 时与 $\mathrm{C}$ 组有显著 性差异 $(P<0.05)$ 。

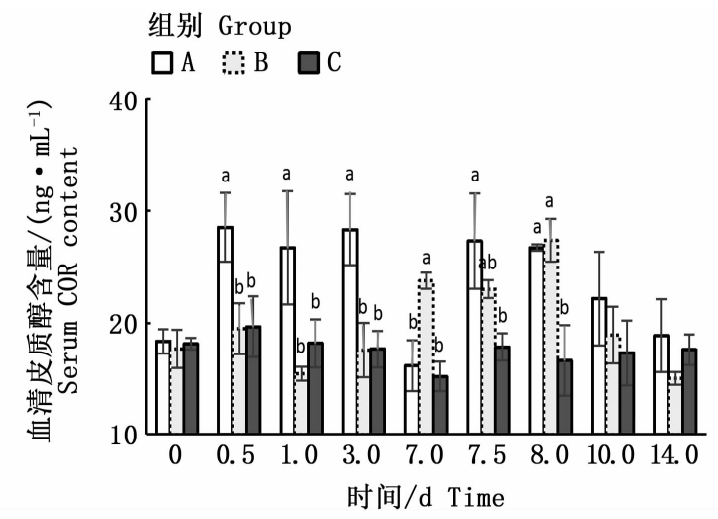

图 2 盐度骤变对花鲇血清皮质醇含量的影响

Fig. 2 Impact of abrupt salinity changes on cortisol content in serum of Lateolabrax maculatus

注: 不同字母表示同一时间不同组间差异显著 $(P<0.05)$

Note: Different letters indicate significant differences between different groups at the same time $(P<0.05)$

\section{4 盐度骤变对花鲇肝脏代谢酶活力的影响}

如图 3 所示, A-I 阶段中, A 组花鲇肝脏中 $\mathrm{AKP}$ 活力先升后降, 在 $0.5 \sim 1 \mathrm{~d}$ 时与 $\mathrm{C}$ 组间有显 著性差异 $(P<0.05)$; ACP 活力先降后升,在 0.5 $\mathrm{d}$ 时与 $\mathrm{C}$ 组有显著性差异 $(P<0.05)$; GPT 活力 呈先升后降的变化, 在 $0.5 \sim 1 \mathrm{~d}$ 时显著高于 $\mathrm{C}$ 组 $(P<0.05)$, 而 $3 \sim 7 \mathrm{~d}$ 时又显著低于 $\mathrm{C}$ 组 $(P<$ $0.05)$; GOT 活力持续高于 $\mathrm{C}$ 组, 但仅 $0.5 \mathrm{~d}$ 时有 显著性差异 $(P<0.05)$ 。在 A-II 阶段中, A 组 $\mathrm{AKP}$ 活力持续升高, 且在 $1 \sim 7 \mathrm{~d}$ 时均与 $\mathrm{C}$ 组有显 著性差异 $(P<0.05)$; $\mathrm{ACP}$ 活力在 $7 \mathrm{~d}$ 时升高至
与 $\mathrm{C}$ 组有显著性差异 $(P<0.05)$; GPT 活力整个 阶段与 $\mathrm{C}$ 组无显著性差异 $(P>0.05)$; GOT 活力 在A-II 阶段均与 $\mathrm{C}$ 组间有显著性差异 $(P<$ $0.05)$ 。

B-I 阶段中, B 组 AKP 活力与 $\mathrm{C}$ 组无显著性 差异 $(P>0.05) ; \mathrm{ACP}$ 活力随时间延长呈波动变 化, 在 $1 、 7 \mathrm{~d}$ 时与 $\mathrm{C}$ 组间有显著性差异 $(P<$ $0.05)$ 。GPT 活力在 $0.5 \sim 1 \mathrm{~d}$ 时与 $\mathrm{C}$ 组有显著性 差异 $(P<0.05)$, 在 $3 \mathrm{~d}$ 时恢复至与 $\mathrm{C}$ 组间无显 著性差异 $(P>0.05)$, 在 $7 \mathrm{~d}$ 时下降至显著低于 $\mathrm{C}$ 组 $(P<0.05)$; GOT 活力持续上升, $0.5 \sim 7 \mathrm{~d}$ 均 与 $\mathrm{C}$ 组间有显著性差异 $(P<0.05)$ 。在 $\mathrm{B}-\mathrm{II}$ 阶段 中, $\mathrm{B}$ 组 $\mathrm{AKP}$ 活力在 $3 \mathrm{~d}$ 时与 $\mathrm{C}$ 组有显著性差异 $(P<0.05)$; 除了 GPT 在 $0.5 \mathrm{~d}$ 时显著低于 $\mathrm{C}$ 组 $(P<0.05)$, 整个阶段 ACP、GPT 和 GOT 活力与 $\mathrm{C}$ 组差异均不显著 $(P>0.05)$ 。

\section{5 盐度骤变对花鲇肝脏抗氧化酶活力的影响}

如图 4 所示, A-I 阶段中, A 组花鲇肝脏中 $\mathrm{SOD}$ 活力在 $0.5 \sim 1 \mathrm{~d}$ 时升高, 与 $\mathrm{C}$ 组之间呈显著 性差异 $(P<0.05)$, 在 $3 \sim 7 \mathrm{~d}$ 下降至与 $\mathrm{C}$ 组无显 著性差异 $(P>0.05)$; GSH-Px 活力随时间延长出 现波动, 仅 $3 \mathrm{~d}$ 时显著高于 $\mathrm{C}$ 组 $(P<0.05)$; $\mathrm{CAT}$ 活力也呈波动变化, 但与 $\mathrm{C}$ 组无显著性差异 $(P>$ $0.05)$; 在 A-II 阶段中, A 组 SOD 活力与 $\mathrm{C}$ 组间无 显著性差异 $(P>0.05)$; GSH-Px 和 CAT 活力均 在 $7 \mathrm{~d}$ 时升高, 与 $\mathrm{C}$ 组间有显著性差异 $(P<0.05)$ 。

B-I 阶段中, SOD 活力变化不大, 与 $\mathrm{C}$ 组之间 无显著性差异 $(P>0.05)$; 而 GSH-Px 随时间延 长呈波动上升变化, 从 $0.5 \mathrm{~d}$ 起即与 $\mathrm{C}$ 组间有显 著性差异 $(P<0.05)$; CAT 活力在 $1 \sim 3 \mathrm{~d}$ 与 $\mathrm{C}$ 组 间有显著性差异 $(P<0.05)$ 。B-II 阶段中, SOD 活力随时间延长呈波动式升高变化, $0.5 、 3 、 7 \mathrm{~d}$ 时与 $\mathrm{C}$ 组有显著性差异 $(P<0.05)$; GSH-Px 活力 则呈下降变化, 在处理 $1 \mathrm{~d}$ 后即下降至 $\mathrm{C}$ 组水平 $(P>0.05)$; CAT 活力先降后升, 仅处理后 $1 \mathrm{~d}$ 与 $\mathrm{C}$ 组间有显著性差异 $(P<0.05)$, 其他时间均与 $\mathrm{C}$ 组间无显著性差异 $(P>0.05)$ 。

\section{3 讨论}

\section{1 盐度骤变对花鲇活动和摄食的影响}

已有的研究和实际养殖生产均已证实, 花鲇 对盐度的适应范围跨度很大。杜涛等 ${ }^{[1]}$ 认为, 盐度 $16 \sim 17$ 是花鲇的最适生长盐度; 刘阳等 ${ }^{[21]}$ 

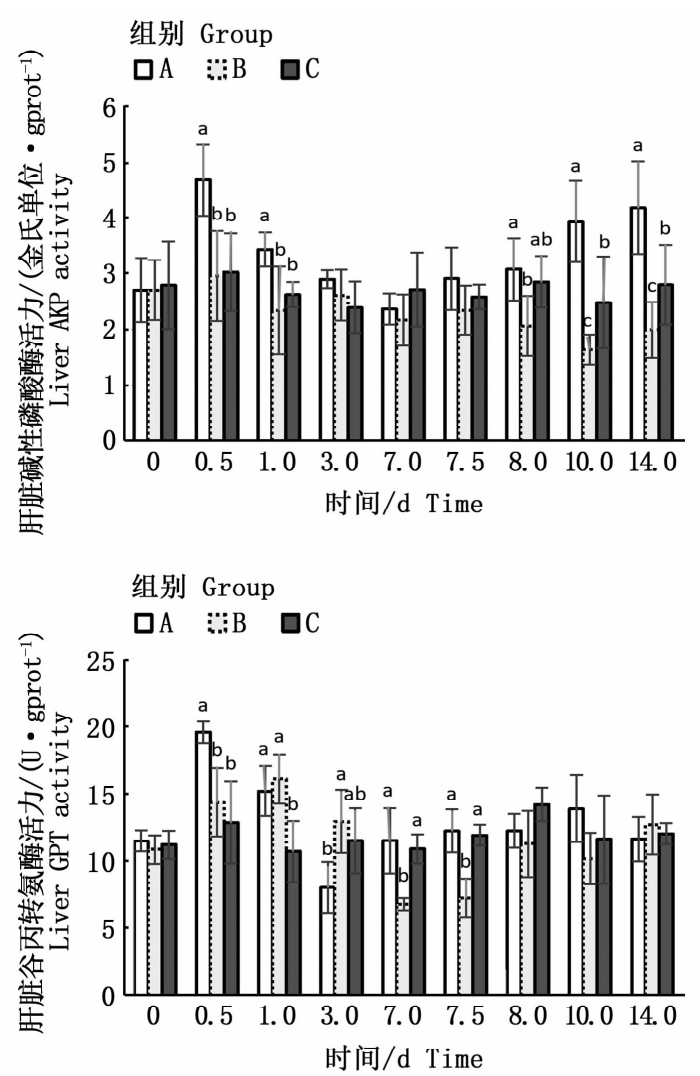
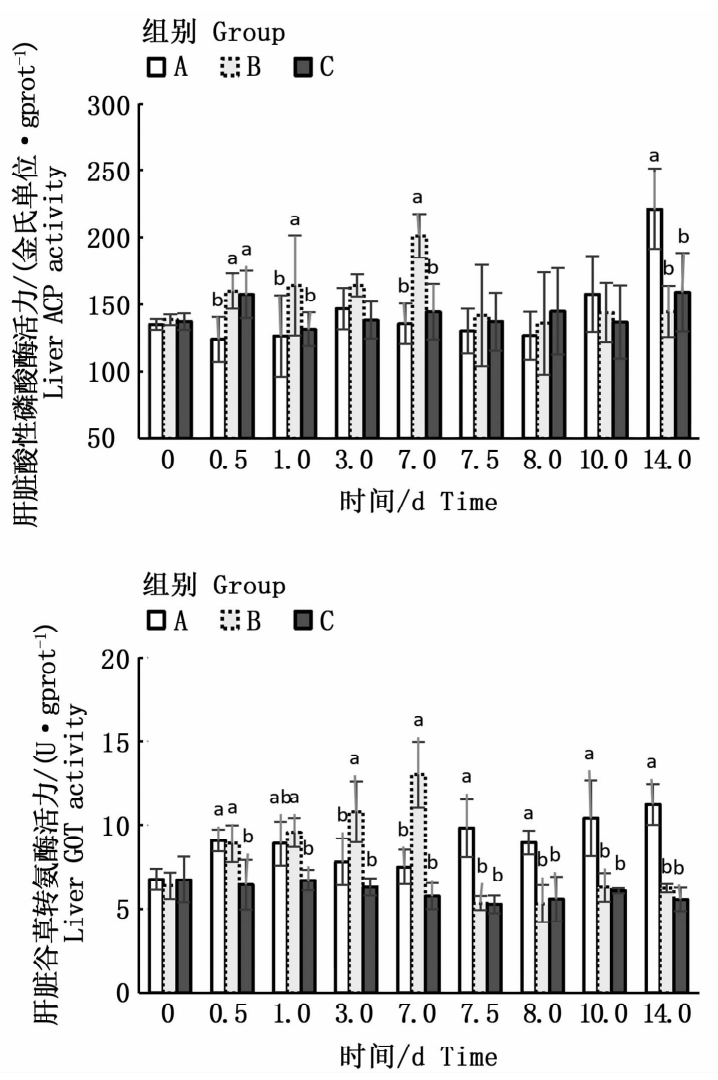

图 3 盐度骤变对花鲇肝脏代谢酶活力的影响

Fig. 3 Impact of abrupt salinity changes on activity of metabolic enzymes in liver of Lateolabrax maculatus 注:不同字母表示同一时间不同组间差异显著 $(P<0.05)$

Note: Different letters indicate significant differences between different groups at the same time $(P<0.05)$

认为, 低盐度环境更有利于花鲇的生长; 温久福 等 ${ }^{[25]}$ 发现, 5 个盐度梯度下 $(0 、 10 、 15 、 20$ 和 $30)$, 仅盐度 30 出现死亡个例。本实验研究了盐 度骤变对花鲇活动和摄食的影响, 虽然盐度骤变 未造成花鲇死亡, 但对其摄食和活动状态仍产生 了一定影响。盐度从 18 骤变到 0 或 33 会造成花 鲇的停食,而且低盐向高盐骤变比高盐向低盐骤 变对鱼活动和摄食的影响更大。从盐度 0 骤变 到 33 或从盐度 33 骤变到 0 , 不仅造成了停食, 而 且使其活动状态也发生了变化, 但经过 $7 \mathrm{~d}$ 的适 应, 能恢复至正常的活动状态。结果表明, 在本 实验条件下, 花鲇对盐度大幅度骤变具有一定的 耐受性和适应性; 相对盐度骤升, 本实验规格的 花鲇对盐度骤降具有更好的适应性。

\section{2 代谢酶活力对盐度骤变的响应}

鱼类在盐度胁迫下, 一般通过加快新陈代谢 来为渗透调节提供能量。此时机体内大量营养 物质被调动, 多种氨基酸参与其中 ${ }^{[26]}$ 。有研究证 明, 体内游离氨基酸如谷氨酸、丙氨酸、甘氨酸、
脯氨酸和牛磺酸的含量会因盐度胁迫而升高 ${ }^{[7]}$ 。 而机体部分的新陈代谢活动是由物质的磷酸化 和去磷酸化来进行调节, 这些过程主要依靠不同 的磷酸酶催化完成 ${ }^{[6]}$ 。已有研究显示, 海水鱼类 在盐度胁迫过程中, 肝脏 AKP 与 ACP 活力会随 着盐度的降低显著升高或者先降低再升高 ${ }^{[7]}$ 。 本研究发现, 血清中磷酸酶比肝脏中的磷酸酶对 盐度骤变的反应更灵敏; 花鲇 AKP 对盐度骤变 的响应特征与其他学者研究结果相似 ${ }^{[7,27]}$; AKP 与 ACP 相比, 对盐度骤变更敏感, 表明其在磷酸 化过程中具有更重要的作用。

一般认为, 转氨酶主要存在肝脏中, 而血清 中的转氨酶活力较低。通过检测血清或肝脏中 转氨酶活力, 可以判断机体的应激程度、评价其 摄食水平和生长发育 ${ }^{[28]}$ 。本实验中, 血清转氨酶 活力为 $1.18 \sim 9.30 \mathrm{U} \cdot \mathrm{L}^{-1}$, 而肝脏转氨酶活力 则是4.25 20.40 U - gprot ${ }^{-1}$, 若忽略血清的比 重, 肝脏中的酶活力是血清中的千倍以上。王涛 等 ${ }^{[29]}$ 发现大黄鱼 (Pseudosciaena crocea) 在盐度突 

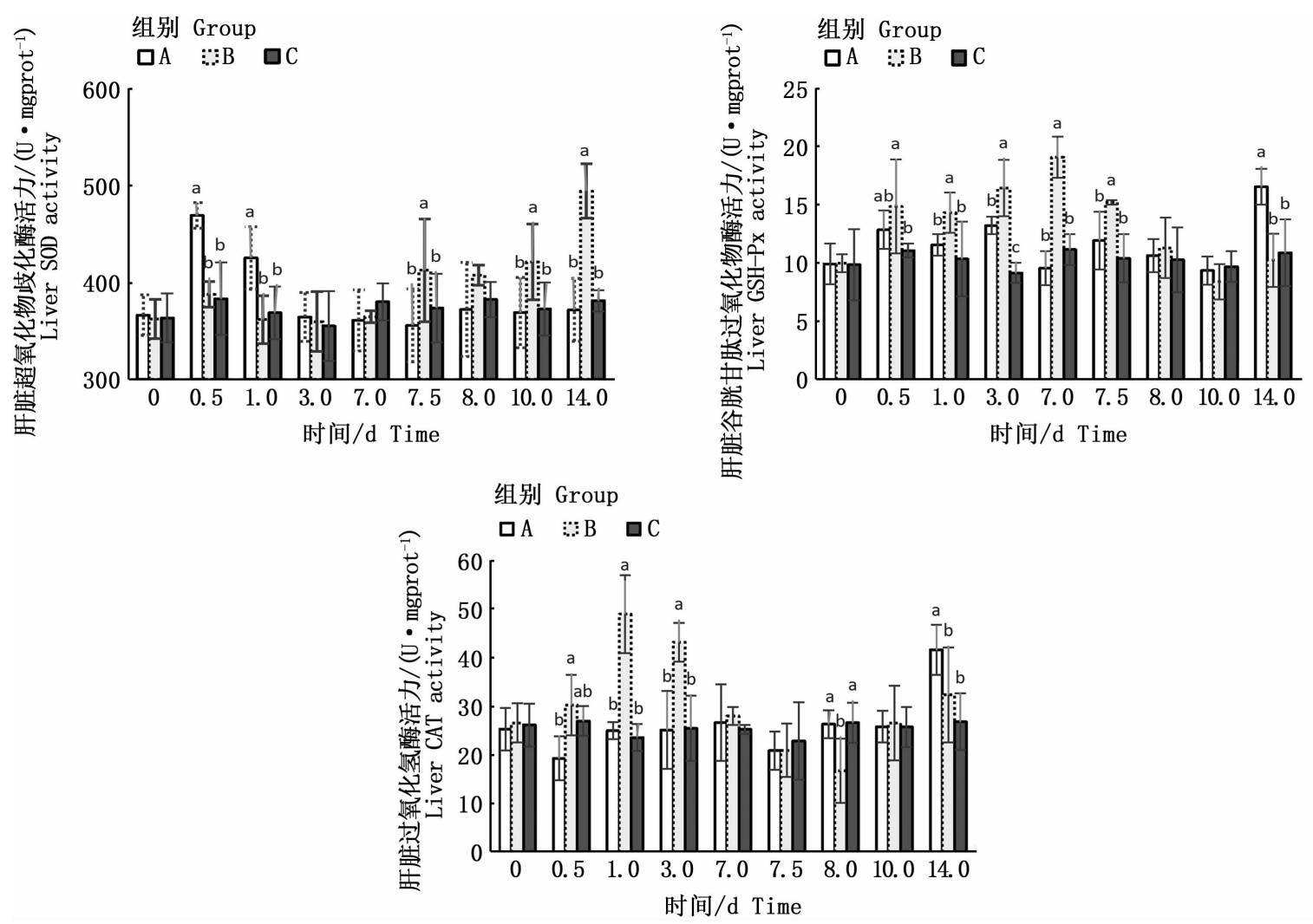

图 4 盐度骤变对花鲇肝脏抗氧化酶活力的影响

Fig. 4 Impact of abrupt salinity changes on activity of antioxidant enzymes in liver of Lateolabrax maculatus 注:不同字母表示同一时间不同组间差异显著 $(P<0.05)$

Note: Different letters indicate significant differences between different groups at the same time $(P<0.05)$

降后血清中 GPT 和 GOT 活力上升, 本研究结果与 其相似。本研究还表明, 盐度不论骤升还是骤 降,均对花鲇形成了胁迫,使其发生应激反应, 导 致转氨酶活力上升。当鱼体适应了环境变化后, 转氨酶活力下降。

\section{3 皮质醇含量对盐度骤变的响应}

处于应激状态下的鱼类,通过下丘脑一垂体 一肾间组织产生皮质醇等类固醇, 并释放到血液 中,体内的 COR 含量在数小时内会急剧升高 ${ }^{[30]}$ 。 COR 通过对糖代谢的调控等形式来提供抵御环 境胁迫所需的能量。但 COR 含量过高或长期维 持在较高的水平, 会对鱼体造成负面影响 ${ }^{[31]}$ 。本 研究显示, 盐度从 18 骤降到 0 时, $0.5 \mathrm{~d}$ 后皮质醇 即有显著变化; 而盐度从 18 骤升到 33 时, $7 \mathrm{~d}$ 后 皮质醇才有显著性变化。可见,花鲇对盐度骤降 可以作出更快的响应。

\section{4 抗氧化酶活力对盐度骤变的响应}

有关环境条件改变会引起生物体内活性氧 自由基产生的现象已有报道 ${ }^{[32-33]}$, 机体为抵御
环境胁迫会作出免疫反应, 产生过量的自由基, 为抵御活性氧自由基对细胞的损伤,机体会通过 抗氧化酶系统来清除体内自由基和活性氧物 质 ${ }^{[34]}$ 。本实验中, SOD 活力在盐度骤降过程中出 现升高, 这与以往研究得出的结果相似 ${ }^{[33,35]}$; 而 GSH-Px 和 CAT 活力在盐度骤降过程中与对照组 相比均无显著性差异 $(P>0.05)$ 。盐度骤升条件 下, SOD 活力未出现上升, 而 GSH-Px 和 CAT 活 力均出现上升。本实验结果表明, SOD 主要在盐 度骤降时发挥作用, 而 GSH-Px 和 CAT 则在盐度 骤升时发挥作用。

一般认为,在清除活性氧的过程中, SOD 最 早发挥作用, 其通过歧化作用来分解超氧阴离子 自由基 $\cdot \mathrm{O}^{-2}$, 并产生 $\mathrm{H}_{2} \mathrm{O}_{2}{ }^{[4]}$ 。再由 CAT 和 GSH-Px 将 $\mathrm{H}_{2} \mathrm{O}_{2}$ 转化为 $\mathrm{H}_{2} \mathrm{O}$ 和 $\mathrm{O}_{2}{ }^{[36]}$ 。本实验 中,盐度从 18 骤降至 0 时, SOD 活力在 $0.5 \mathrm{~d}$ 时 显著升高, GSH-Px 则在 $3 \mathrm{~d}$ 时达到峰值; 盐度从 33 骤降至 0 时, SOD 活力持续升高, GSH-Px 和 CAT 活力则在实验过程后期中出现上升,但与对 
照组相比无显著性差异。这种变化规律可能印 证了 SOD 在活性氧清除反应中处于核心的地 位 $^{[37]}$ 。SOD、GSH-Px 和 CAT 在抗氧化系统中相 互协调,发挥着清除自由基的功能 ${ }^{[35]}$ 。

\section{4 小结}

花鲇在本实验盐度大幅度骤变条件下, 体内 代谢酶和抗氧化酶活力以及皮质醇含量都出现 变化, 大部分条件下均呈现随时间延长先升高后 降低的趋势, 表明花鲈在盐度骤变时产生了应激 反应。而后可在 $3 \sim 7 \mathrm{~d}$ 内获得对骤变盐度的适 应, 说明花鲈对盐度变化具有良好的耐受性和适 应性。本实验条件下, 从代谢酶、抗氧化酶活力 和皮质醇含量等变化特征来看, 花鲇对盐度骤降 比盐度骤升有更强适应能力。

\section{参考文献:}

１１～张晨捷,施兆鸿, 王建钢, 等. 盐度影响海水硬骨鱼 类渗透压调节机理的研究与展望 $[\mathrm{J}]$. 海洋渔业, 2013, 35(1): 108 - 116 .

ZHANG C J, SHI Z H, WANG J G, et al. On salinity-related effects on osmoregulation mechanism in marine teleost $[\mathrm{J}]$. Marine Fisheries, 2013, 35 (1) : $108-116$.

[2] CHOI C Y, AN K W, AN M I. Molecular characterization and mRNA expression of glutathione peroxidase and glutathione S-transferase during osmotic stress in olive flounder (Paralichthys olivaceus ) [ J ]. Comparative Biochemistry and Physiology Part A: Molecular \& Integrative Physiology ,2008,149(3) : $330-337$.

[3] 王艳玲, 赵金良, 赵 岩. 环境胁迫对鱼类免疫机 制的影响研究进展 [J]. 河北渔业, 2020,44 (5) : $46-50,62$.

WANG Y L, ZHAO J L, ZHAO Y. Research progress on the effect of environmental stress on the immune mechanism of fish $[\mathrm{J}]$. Hebei Fisheries, 2020,44 (5): 5 ) 46 - 50,62.

[4] 连灵君. 用多项生物标志物评价铅对小鼠的氧化 损伤 [D]. 杭州: 浙江大学医学院,2006.

LIAN L J. The evaluation of using a number of biomarkers on oxidative damage in mice by lead [D ]. Hangzhou: Medical College of Zhejiang University, 2006.

[5] DA SILVA A Z, ZANETTE J, FERREIRA J E, et al. Effects of salinity on biomarker responses in
Crassostrea rhizophorae ( Mollusca, Bivalvia) exposed to diesel oil $[\mathrm{J}]$. Ecotoxicology and Environmental Safety, 2005, 62(3) : 376 - 382 .

[6]王 卓, 么宗利, 林听听, 等. 碳酸盐碱度对青 海湖裸鲤幼鱼肝和肾 SOD、ACP 和 AKP 酶活性的 影响 $[\mathrm{J}]$. 中国水产科学, 2013,20 (6) : 1212 1218.

WANG Z, YAO Z L, LIN T T, et al. Effects of carbonate alkalinity stress on SOD, ACP, and AKP activities in the liver and kidney of juvenile Gymnocypris przewalskii [ J ]. Journal of Fishery Sciences of China,2013,20(6) : 1212 - 1218 .

[7] 施兆鸿, 廖雅丽, 王孝杉, 等. 盐度骤降对云纹石斑 鱼肝脏代谢酶活力的影响 [J]. 海洋渔业, 2016, $38(2)$ : $174-182$.

SHI Z H, LIAO Y L, WANG X S, et al. Impact of abrupt salinity decrease on metabolic enzymes in the liver of Epinehelus moara $[\mathrm{J}]$. Marine Fisheries, 2016, 38(2) : $174-182$.

[8] SAMSONOVA M V, MINKOVA N O,LAPTEVA TI, et al. Aspartate and alanine aminotransferases in early development of the keta $[\mathrm{J}]$. Russian Journal of Developmental Biology, 2003, 34(1) :14 - 18 .

［9］马 慧, 柳淑芳, 马 骞, 等. 硬骨鱼类渗透压 的内分泌激素调节机制 [J]. 水产科学, 2012,31 (1) : $55-59$.

MA H, LIU S F, MA Q, et al. Endocrine hormone adjustment mechanism of osmotic pressure in teleost [J]. Fisheries Science, 2012, 31 (1) :55 - 59 .

[10］雷雯霖. 海水鱼类养殖理论与技术 $[\mathrm{M}]$. 北京: 中 国农业出版社 2005: $745-774$.

LEI J L. Marine fish culture theory and techniques [M]. Beijing: China Agriculture Press, 2005: 745 -774 .

[11] 王永新, 陈建国, 孙帼英. 温度和盐度对花鲇肧 胎及前期仔鱼影响的初步报告 $[\mathrm{J}]$. 水产科技情 报, 1995, 22(2):54-57.

WANG Y X, CHEN J G, SUN G Y. Effects of temperature and salinity on embryo and early larvae development in common sea bass, Lateolabarx japonieus $[\mathrm{J}]$. Fisheries Science \& Technology Information, 1995, 22(2):54-57.

[12] 叶金聪. 温、盐度对鲈鱼早期仔鱼生长及存活率 的影响 $[\mathrm{J}]$. 福建水产, 1997, 11(1):14-18.

YE J C. Effects of temperature and salinity on growth and survival rate of pre-larvae Japanese sea bass, Lateolabarx japonieus [ J ]. Journal of Fujian Fisheries, 1997, 11(1):14-18. 
[13］王 艳, 胡先成, 罗 颖. 盐度对鲇鱼稚鱼的生长 及酸组成的影响 $[\mathrm{J}]$. 重庆师范大学学报 ( 自然科 学版), 2007, 24(2):62-65.

WANG Y, HU X C, LUO Y. Effects of salinity on growth and fatty acids composition of juvenile Lateolabarx japonieus $[\mathrm{J}]$. Journal of Chongqing Normal University (Natural Science Edition), 2007 , $24(2): 62-65$.

[14]王 艳, 胡先成, 韩 强. 不同盐度条件下饥饿及 恢复摄食鲈稚鱼脂肪酸的组成 $[\mathrm{J}]$. 水产科学, 2008,27 ( 7 ) :334 - 339.

WANG Y, HU X C, HAN Q. Composition of fatty acids in starved and refeeding juvenile sea perch Lateolabrax japonicas under different salinities $[\mathrm{J}]$.

Fisheries Science, 2008, 27(7):334-339.

[15] 王 艳. 低盐胁迫对鲇鱼稚鱼生长及生理生化指 标的影响 [D]. 重庆: 重庆师范大学, 2008.

WANG Y. Effects of low-salt stress on the growth, physiological and biochemical indexes of juvenile Lateolabrax japoncus [D ]. Chongqing: Chongqing Normal University, 2008.

［16］王 艳, 胡先成. 不同盐度下鲇鱼稚鱼鳃的显微 结构观察 $[\mathrm{J}]$. 海洋科学, 2009, 33 (12) : $138-$ 142.

WANG Y, HU X C. Microscopical observation on the gill structure of juvenile Lateolabrax japonicus under different salinities $[\mathrm{J}]$. Marine Sciences, 2009, 33(12):138-142.

[17] 杜 涛, 黄 洋, 覃雪迎, 等. 不同盐度养殖 1 龄 花鲇 (Lateolabrax maculatus) 的生长特性差异分析 [J]. 海洋与湖沼, 2013, 44(2):337-341.

DU T, HUANG Y, QIN X Y, et al. Difference analysis on growth characteristic of one year old Lateolabrax maculatus cultured at different salinity [J]. Oceanologia et Limnologia Sinica, 2013, 44 (2) :337 - 341 .

[18］张 沛, 迟美丽, 温海深, 等. 不盐度调控对花鲇 (Lateolabrax maculatus) 生长相关基因表达的影响 [J]. 海洋与湖沼, 2015, 46(2) :446 - 453 .

ZHANG P, CHI M L, WEN H S, et al. Cloning of growth hormone receptor and salinity effects on the expression of its related genes in sea bass Lateolabrax maculatus $[\mathrm{J}]$. Oceanologia et Limnologia Sinica, $2015,46(2): 446-453$.

[19］王海亮, 温海深, 张晓燕. 盐度胁迫对花鲈幼鱼 肠道抗氧化和非牧民性免疫能力的影响 $[\mathrm{J}]$. 现代 农业科技, 2016(4) : 261 -263.

WANG H L, WEN H S, ZHANG X Y. Effects of salinity stress on antioxidant enzyme and non-specific immunity activities in the intestine of juvenile Lateolabrax maculatus [ J ]. Modern Agricultural Science and Technology, 2016(4) : $261-263$.

[20] 韩 枫, 黄杰斯, 温海深, 等. 不同盐度条件下亚 硝酸态氮与非离子氨对花鲈幼鱼的毒性实验 $[\mathrm{J}]$. 海洋科学, 2016,40 (10): $7-14$.

HAN F, HUANG J S, WEN H S, et al. Toxicity of unionized ammonia and nitrite on juvenile Lateolabrax maculatus according to salinity $[\mathrm{J}]$. Marine Sciences, 2016,40 (10):7 - 14 .

[21] 刘 阳, 温海深, 李吉方, 等. 不同盐度与雌二醇 投喂对花鲈幼鱼生长性能的影响 $[\mathrm{J}]$. 海洋科学, 2017, 41(6):8-16.

LIU Y, WEN H S, LI J F, et al. Effects of different salinities and estradiol feeding on growth performance of Lateolabrax maculatus juveniles [ J ]. Marine Sciences, 2017, 41(6):8-16.

[22] 刘阳, 温海深, 李吉方, 等. 盐度与 $\mathrm{pH}$ 对花鲇孵 化、初孵仔鱼成活及早期幼鱼生长性能的影响 [J]. 水产学报, 2017, 41 (12):1867 - 1876 .

LIU Y, WEN H S, LI J F, et al. Effects of salinity and $\mathrm{pH}$ on hatching and larval activity of Lateolabrax maculatus $[\mathrm{J}]$. Journal of Fisheries of China, 2017, 41 ( 12 ): 1867 - 1876.

[23] 张晓燕, 温海深, 张凯强, 等. 花鲇等渗点分析及 海水淡化对 $\mathrm{Na}^{+} / \mathrm{K}^{+} / \mathrm{Cl}^{-}$浓度、 $\mathrm{Na}^{+}-\mathrm{K}^{+}$-ATP 酶 活性及基因表达的影响 $[\mathrm{J}]$. 水产学报, 2018, 42 (8) : $1199-1208$.

ZHANG X Y, WEN H S, ZHANG K Q, et al. Analysis of the isotonic point and effects of seawater desalination on the $\mathrm{Na}^{+} / \mathrm{K}^{+} / \mathrm{Cl}^{-}$concentration, $\mathrm{Na}^{+}-\mathrm{K}^{+}$-ATPase activity and relative gene expressions in Lateolabrax maculatus $[\mathrm{J}]$. Journal of Fisheries of China, 2018, 42(8) :1199-1208.

[24] 温久福, 蓝军南, 周 慧, 等. 盐度对花鲇幼鱼消 化酶和抗氧化系统的影响 $[\mathrm{J}]$. 动物学杂志, 2019, 54(5):719-726.

WEN J F, LAN J N, ZHOU H, et al. Effects of salinity on digestive enzymes and antioxidant system of juvenile Lateolabrax maculatus $[\mathrm{J}]$. Chinese Journal of Zoology, 2019, 54(5) : 719 - 726.

[25] 温久福, 蓝军南, 曹 明, 等. 盐度对花鲈幼鱼 鳃、脾及肌肉组织结构的影响 $[\mathrm{J}]$. 渔业科学进 展, 2020, 41 (1):112-118.

WEN J F, LAN J N, CAO M, et al. Effects of salinity on the histological structure of the gills, spleen, and muscle in Lateolabrax maculatus 
juveniles $[\mathrm{J}]$. Progress in Fishery Sciences, 2020, $41(1): 112-118$.

[26] CUZON G, LAWRENCEB A, GAXIOLAC G, et al. Nutrition of Litopenaeus vannamei reared in tanks or in ponds $[\mathrm{J}]$. Aquaculture, $2004,235(1-4)$ :513 551.

[27] 支兵杰, 刘 伟, 赵春刚, 等. 盐度对大麻哈鱼幼 鱼消化酶及碱性磷酸酶活力的影响 $[\mathrm{J}]$. 上海海 洋大学学报, 2009, 18(3):289-294.

ZHI B J, LIU W, ZHAO C G, et al. Effects of salinity on digestive enzyme and alkaline phosphatase activity of young chum salmon (Oncorhynchus keta Walbaum ) [ J ]. Journal of Shanghai Ocean University, 2009, 18(3) :289-294.

[28] SAMSONOVA M V, MINKOVA N O, LAPTEVA T $\mathrm{I}$, et al. Aspartate and alanine aminotransferases in early development of the $\operatorname{keta}[\mathrm{J}]$. Russian Journal of Developmental Biology, 2003, 34(1) :14 - 18

[29］王 涛, 苗 亮, 李明云, 等. 突降盐度胁迫对大 黄鱼 (Pseudosciaena crocea) 血清生理生化及鳃丝 $\mathrm{Na}^{+} / \mathrm{K}^{+}$-ATP 酶活性的影响 $[\mathrm{J}]$. 海洋与湖沼, $2013,44(2): 421-426$.

WANG T, MIAO L, LI M Y, et al. Effects of abrupt salinity decrease on serum physiology, biochemistry and gill $\mathrm{Na}^{+} / \mathrm{K}^{+}$-ATPase activity of the large yellow croaker Pseudosciaena crocea $[\mathrm{J}]$. Oceanologia et Limnologia Sinica, 2013, 44(2):421 - 426.

[30］施兆鸿, 谢明媚, 彭士明, 等. 温度胁迫对银鲳 (Pampus argenteus) 幼鱼消化酶活性及血清生化指 标的影响 $[\mathrm{J}]$. 渔业科学进展, 2016,37(5) : 30 37.

SHI Z H, XIE M M, PENG S M, et al. Effects of temperature stress on activities of digestive enzymes and serum biochemical indices of Pampus argenteus juveniles $[\mathrm{J}]$. Progress in Fishery Sciences, 2016, $37(5): 30-37$.

［31］舟风霞, 金文杰, 黄 屾, 等. 盐度变化对鱼类影 响的研究进展 $[\mathrm{J}]$. 西北农林科技大学学报 (自然 科学版), 2020, 48(8): 10-18.

RAN F X, JIN W J, HUANG S, et al. Research progress on the effects of salinity change on fish $[\mathrm{J}]$. Journal of Northwest Agriculture and Forestry University (Natural Science Edition), 2020, 48(8) :
$10-18$.

[32］唐贤明, 隋 悬, 田景波, 等. 盐度对大菱鲆幼鱼 耗氧率和排氨率的影响 $[\mathrm{J}]$. 南方水产, 2006, 2 (4) : $54-58$.

TANG X M, SUI Z, TIAN J B, et al. Effects of salinity on metabolic rate of juvenile turbot (Scophamus maximus) [J]. South China Fisheries Science, 2006,2 (4) :54 - 58.

［33］尹飞,孙 鹏, 彭士明, 等. 低盐度胁迫对银鲳幼 鱼肝脏抗氧化酶、鳃和肾 ATP 酶活力的影响 $[\mathrm{J}]$. 应用生态学报, 2011,22(4):1059-1066.

YIN F, SUN P, PENG S M, et al. Effects of low salinity stress on the antioxidant enzyme activities in juvenile Pampus argenteus liver and the ATPase activities in its gill and kidney $[\mathrm{J}]$. Chinese Journal of Applied Ecology, 2011,22(4):1059-1066.

[34] 赵 峰, 庄 平, 章龙珍, 等. 施氏鲟不同组织抗 氧化酶对水体盐度升高的响应 $[\mathrm{J}]$. 海洋水产研 究, 2008,29(4) : 65-69.

ZHAO F, ZHUANG P, ZHANG L Z, et al. Responses of antioxidases in different tissues of Acipenser schrenckii to increased salinity in water $[\mathrm{J}]$. Marine Fisheries Research, 2008 ,29(4) : 65 -69.

[35］张晨捷, 张艳亮, 高权新, 等. 低盐胁迫对黄姑鱼幼 鱼肝脏抗氧化功能的影响 $[\mathrm{J}]$. . 南方水产科学, $2015,11(4): 59-64$.

ZHANG C J, ZHANG Y L, GAO Q X, et al. Effects of low salinity stress on antioxidant function in liver of juvenile Nibea albiflora $[\mathrm{J}]$. South China Fisheries Science, $2015,11(4): 59-64$.

[36] 廖雅丽, 张晨捷, 彭士明, 等. 盐度对云纹石斑鱼 抗氧化酶及溶菌酶活性的影响 $[\mathrm{J}]$. 上海海洋大 学学报,2016, 25(2): $167-176$.

LIAO Y L, ZHANG C J, PENG S M, et al. Effects of salinity on activities of liver antioxidant enzymes and plasmalysozyme of Epinehelus moara [ J ]. Journal of Shanghai Ocean University, 2016, 25 (2) $: 167-176$.

[37 ] MARTNEZ-ALVAREZ R M, HIDALGO M C, DOMEZAIN A, et al. Physiological changes of sturgeon Acipenser naccarii caused by increasing environmental salinity [J]. Journal of Experimental Biology, 2002, 205 (23) :3699-3706. 


\title{
Impact of abrupt salinity changes on activitiy of metabolic enzymes, antioxidant enzymes and cortisol content in serum and liver of Lateolabrax maculatus
}

\author{
WANG Xiaoshan ${ }^{1}$, FANG Xiu ${ }^{2}$, PENG Shiming ${ }^{1}$, WANG Jing ${ }^{2}$, SHI Zhaohong ${ }^{1}$ \\ (1. East China Sea Fisheries Research Institute, Chinese Academy of Fishery Sciences, Shanghai 200090; \\ 2. Fujian Minwell Industrial CO. , LTD. , Fuding Fujian 355200)
}

\begin{abstract}
Lateolabrax maculatus is an important economic fish species in China. It is a eurysaline species and can survive and grow in water environment with varieties of salinity. It is of practical significance to study the tolerance and adaptability of $L$. maculatus, because it usually suffers from sudden increase or decrease of salinity in aquaculture environment. This work investigated the effects of abrupt salinity changes on the activitiy of metabolic enzymes and antioxidant enzymes and cortisol content in liver of L. maculatus by simulating salinity changes of water for its breeding. The salinity was reduced from 18 to 0 and remained for 7 $\mathrm{d}$, and then increased to 33 sharply after acclimation and remained for $7 \mathrm{~d}$. Correspondingly, in the other treatment, the salinity increased from 18 to 33 , and then decreased to 0 sharply after acclimation and remained for $7 \mathrm{~d}$. Samples were taken on $0,0.5,1,3,7 \mathrm{~d}$ after the start of the experiment, respectively. The activitiy of alkaline phosphatase, acid phosphatase, glutamic-pyruvic transaminase, glutamic-oxalacetic transaminase, superoxide dismutase, catalase, glutathione peroxidase in serum and liver of L. maculatus was tested, and cortisol (COR) content in serum was monitored as well. Results showed that the abrupt salinity changes would have impact on activitiy of metabolic enzymes and antioxidant enzymes, and COR content of $L$. maculatus. The activitiy of enzymes and COR content showed a trend of increasing firstly and then declining with time prolonging under most situations of abrupt salinity changes. These results showed that L. maculatus had a stress reaction during the abrupt changing of salinity, and could adapt to the change within 3-7 days, which indicated that L. maculatus had a substantial tolerance and adaptability to the abrupt changes in salinity. The changes of morphological characteristics, metabolic enzyme activity, antioxidant enzyme activity and COR content showed that L. maculatus could more rapidly adapt to sudden drop in salinity and was more adaptable to low-salinity environment.
\end{abstract}

Keywords : Lateolabrax maculatus; abrupt change in salinity; metabolic enzyme activity ; antioxidant enzyme activity; cortisol content 